time periods (trial: 2013-15 and program: 2016-19). For the trajectory analysis, we applied a Poisson model to identify and fit health services to testing groups.

Results Among the 11 services who participated in both the trial and program, 7871 tests were conducted in total. The median number of tests per month in the trial was 241 (IQR:178-305) and 408(IQR:294-538) in the program, with no significant trend in the trial (5.6 tests per month, $\mathrm{p}=0.190$ ) but a significant increasing trend in the program (10.52 tests per month, $\mathrm{p}<0.001)$. Among the 31 program services ( $n=20,622$ tests), three trajectory group were identified (low, medium, high). There was a significant upward trend in mean monthly testing in the 'high' trajectory group (model-predicted linear regression coefficient:0.03, $\mathrm{p}=0.002$ ).

Conclusions Our findings suggest POC testing can be scaledup and sustained as part of a routinely implemented program, achieving greater than expected testing numbers to support the clinical and public health benefits of more timely treatment. Further research is underway to investigate barriers among the 'low' testing sites. These findings support the proposed transition from syndromic management towards aetiological diagnosis and treatment in low- and middle-income countries.

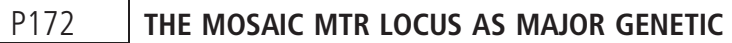 DETERMINANT OF AZITHROMYCIN RESISTANCE OF NEISSERIA GONORRHOEAE, GERMANY, 2018}

\begin{abstract}
${ }^{1} S$ Banhart, ${ }^{2} \mathrm{R}$ Selb*, ${ }^{1} \mathrm{~S}$ Oehlmann, ${ }^{3,4}$ J Bender, ${ }^{5} \mathrm{~S}$ Buder, ${ }^{2} \mathrm{~K}$ Jansen, ${ }^{1} \mathrm{D}$ Heuer. ${ }^{1}$ Unit 'Sexually Transmitted Bacterial Infections', Department for Infectious Diseases, Robert Koch Institute, Berlin, Germany; ${ }^{2}$ Unit 'HIVIAIDS, STI and Blood-borne Infections', Department for Infectious Disease Epidemiology, Robert Koch Institute, Berlin, Germany; ${ }^{3}$ Unit 'Nosocomial Pathogens and Antibiotic Resistances', Department of Infectious Diseases, Robert Koch Institute, Wernigerode, Germany; ${ }^{4}$ European Programme for Public Health Microbiology Training (EUPHEM), European Centre for Disease Prevention and Control (ECDC), Solna, Sweden; ${ }^{5}$ German Reference Laboratory for Gonococci, Unit 'Sexually Transmitted Bacterial Infections', Department for Infectious Diseases, Robert Koch Institute, Berlin, Germany
\end{abstract}

\subsection{6/sextrans-2021-sti.270}

Background Azithromycin resistant Neisseria gonorrhoeae (NG) isolates increased from $4.3 \%$ in 2016 to $9.2 \%$ in 2018 within the German Gonococcal Resistance Network (GORENET) NG sample collection. Using whole genome sequencing (WGS) of NG isolates in combination with clinical and epidemiological data, we aim to understand this observed increase.

Methods GORENET was set up in 2013 as a laboratory network to monitor NG infections in Germany by collection of NG isolates, epidemiological and clinical data. In 2018, isolates with reduced susceptibility to azithromycin (MIC $\geq 0.25$ $\mathrm{mg} / \mathrm{L}$ ) were analyzed by WGS followed by assignment of sequence types based on NG multiantigen sequence typing (NG-MAST) and multilocus sequence typing (MLST), detection of antimicrobial resistance determinants and generation of a core SNP distance-based neighbor-joining phylogenetic tree. Comparison with published isolates was performed based on a custom ad-hoc cgMLST scheme and calculation of a minimum spanning tree.

Results Whole genome phylogenetic analyses resulted in 4 major clades corresponding to NG-MAST genogroups G2400, G3779 (G1407), G5441 and G12302. The clade comprising G12302 accounted for the majority of isolates with azithromycin resistance $(\mathrm{MIC}>0.5 \mathrm{mg} / \mathrm{L})$ and was characterized by the presence of the recently described Neisseria lactamica-like mosaic mtr locus. In addition, strains in this clade were significantly associated with rectal infection site and younger age. Comparison with published isolates revealed similarity between a US and a German isolate of MLST ST9363 (12 nucleotides difference) and between a US and a German isolate of MLST ST11422 (21 nucleotides difference).

Conclusion Our data indicate the recently observed increase in isolates resistant to azithromycin in Germany coincides with clonal expansion of NG-MAST genogroup G12302 and suggest that, together with horizontal gene transfer of resistance determinants and well-established point mutations, international spread of resistant lineages plays a major role regarding azithromycin resistance in Germany.

\section{P173 SEXUALLY TRANSMITTED INFECTION DIAGNOSES AMONG HIV PRE-EXPOSURE PROPHYLAXIS USERS LARGELY EXPLAINED BY PARTNER NUMBERS AND TESTING FREQUENCY}

${ }^{1} \mathrm{U}$ Marcus*, 'S Schink, ${ }^{2} \mathrm{~J}$ Maaß, ${ }^{3} \mathrm{C}$ Weber. ${ }^{1}$ Robert Koch Institute, Berlin, Germany; ${ }^{2}$ CheckPoint Hannover, Hannover, Germany; ${ }^{3}$ Checkpoint Berlin, Berlin, Germany

\subsection{6/sextrans-2021-sti.271}

Background There are conflicting data on the impact of increasing HIV Pre-exposure prophylaxis (PrEP) use on transmission and diagnosis of other sexually transmitted infections (STI) such as syphilis, gonorrhoea and chlamydia infection. We used observational data from German HIV/STI checkpoints on visits of PrEP users and non-users in 2019 and 2020 to analyse determinants for STI diagnosis.

Methods Checkpoint clients fill out an online questionnaire at each visit. Demographic and behavioural data of the online questionnaire are linked to laboratory results. We analysed data of client-subgroups with relevant PrEP use (at least 1\% of visits in the group by people using PrEP) for determinants of STI diagnoses using univariate and multivariate logistic regression models. PrEP use was categorized as never; intended; former; on demand, and regular.

Results Data from 12,235 checkpoint visits including blood testing and 10,131 checkpoint visits including additional swabtesting for gonorrhoea and chlamydia could be included in the analysis. Active syphilis was diagnosed 2-3 times more frequently in visits of PrEP users compared to visits of non-users, gonorrhoea and/or chlamydia was diagnosed in 20\% of PrEP user visits and $12 \%$ of non-PrEP-user visits. Three of four regular PrEP users had last been checked for STI within the last 3 months, but only one of nine non-PrEP-users. The number of sex partners and condomless sex partners was higher for PrEP users than for non-PrEP-users. In multivariate regression models, number of sex partners, number of condomless sex partners, and recency of STI testing explained the probability of STI diagnosis, the type of PrEP use was not significant.

Conclusions Differences regarding STI diagnoses between PrEP users and non-users are largely explained by partner numbers and testing frequency. Due to the cross-sectional nature of our data we cannot determine whether initiating PrEP leads to higher partner numbers and/or reduced condom use. 